\title{
Positive stable realizations for fractional descriptor continuous-time linear systems
}

\author{
TADEUSZ KACZOREK
}

\begin{abstract}
A method for computation of positive asymptotically stable realizations of fractional descriptor continuous-time linear systems with regular pencil is proposed. The method is based on the decomposition of the improper transfer matrix into strictly proper matrix and a polynomial matrix. A procedure for decomposition of a positive asymptotically stable realization is given and illustrated by a numerical example.
\end{abstract} tem

Key words: positive, stable, realization, procedure, descriptor, continuous-time, linear sys-

\section{Introduction}

A dynamical system is called positive if its trajectory starting from any nonnegative initial state remains forever in the positive orthant for all nonnegative inputs. An overview of state of the art in positive theory is given in the monographs [5, 13]. Variety of models having positive behavior can be found in engineering, economics, social sciences, biology and medicine, etc. The positive fractional linear systems have been addressed in [10, 11, 22, 25].

An overview on the positive realization problem is given in $[1,5,13,14]$. The realization problem for positive continuous-time and discrete-time linear systems has been considered in $[6,7,15-17,19-21]$ and the positive realization problem for discrete-time systems with delays in [20-21]. The realization problem for fractional linear systems has been analyzed in $[15,18,22]$ and for positive 2D hybrid systems in [19]. A method based on the similarity transformation of the standard realizations to the desired form has been proposed in [17].

Positive stable realizations problem for continuous-time standard and fractional linear systems has been addressed in $[7,15]$ and computation of realizations of discretetime cone systems in [8]. A method for computation of positive realizations of descriptor continuous-time linear systems has been proposed in [23].

The Author is with Bialystok University of Technology, Faculty of Electrical Engineering, Wiejska 45D, 15-351 Bialystok, Poland. E-mail: kaczorek@isep.pw.edu.pl

This work was supported by National Science Centre in Poland under work no. N N514 638940.

Received 28.05.2012. 
Standard descriptor linear systems has been addressed in $[2,12]$ and positive descriptor systems has been analyzed in [3, 4, 9, 27].

In this paper a method for computation of positive asymptotically stable realizations of fractional descriptor continuous-time linear systems with regular pencils will be proposed.

The paper is organized as follows. In section 2 the positive realization problem for standard and descriptor continuous-time linear systems is recalled. The positive fractional linear systems are considered in section 3. The realization problem for positive fractional linear systems is addressed in section 4. Concluding remarks are given in section 5 .

The following notation will be used: $\Re$ - the set of real numbers, $\Re^{n \times m}$ - the set of real matrices, $\Re_{+}^{n \times m}$ - the set of $n \times m$ matrices with nonnegative entries and $\Re_{+}^{n}=\Re_{+}^{n \times 1}$, $\mathfrak{R}^{p \times m}(s)$ - the set of $p \times m$ rational matrices in $s$ with real coefficients, $\mathfrak{R}^{p \times m}[s]$ - the set of $p \times m$ polynomial matrices in $s$ with real coefficients, $M_{n}$ - the set of $n \times n$ Metzler matrices, $I_{n}-$ the $n \times n$ identity matrix.

\section{Preliminaries and positive realization problem for standard and descriptor linear systems}

Consider the standard continuous-time linear system

$$
\begin{aligned}
& \dot{x}(t)=A x(t)+B u(t) \\
& y(t)=C x(t)+D u(t)
\end{aligned}
$$

where $x(t) \in \mathfrak{R}^{n}, u(t) \in \mathfrak{R}^{m}, y(t) \in \mathfrak{R}^{p}$ are the state, input and output vectors and $A \in \mathfrak{R}^{n \times n}, B \in \mathfrak{R}^{n \times m}, C \in \mathfrak{R}^{p \times n}, D \in \mathfrak{R}^{p \times m}$.

Definition 1 [13] The system (1) is called the (internally) positive if $x(t) \in \Re_{+}^{n}$, $y(t) \in \mathfrak{R}_{+}^{p}, t \geqslant 0$ for any initial conditions $x(0)=x_{0} \in \mathfrak{R}_{+}^{n}$ and all inputs $u(t) \in \mathfrak{R}_{+}^{m}$, $t \geqslant 0$.

Theorem $1[5,13]$ The system (1) is positive if and only if

$$
A \in M_{n}, B \in \mathfrak{R}_{+}^{n \times m}, \quad C \in \mathfrak{R}_{+}^{p \times n}, \quad D \in \mathfrak{R}_{+}^{p \times m} .
$$

The transfer matrix of the system (1) is given by

$$
T(s)=C\left[I_{n} s-A\right]^{-1} B+D \in \Re^{p \times m}(s) .
$$

The transfer matrix $T(s) \in \mathfrak{R}^{p \times m}(s)$ is called proper if and only if

$$
\lim _{s \rightarrow \infty} T(s)=K \in \mathfrak{R}^{p \times m}
$$


and it is called strictly proper if $K=0$. Otherwise the transfer matrix is called improper.

Definition 2 Matrices (2) are called a positive realization of transfer matrix $T(s)$ if they satisfy the equality (3). The realization is called positive stable if the matrix A of (2) is an asymptotically stable Metzler matrix.

Theorem $2[5,13]$ The positive system (1a) (or the Metzler matrix $A \in M_{n}$ ) is asymptotically stable if and only if the coefficients of the polynomial

$$
\operatorname{det}\left[I_{n} s-A\right]=s^{n}+a_{n-1} s^{n-1}+\ldots+a_{1} s+a_{0}
$$

are positive, i.e. $a_{k}>0$ for $k=0,2, \ldots, n-1$.

Other asymptotic stability tests of positive linear systems are given in [13, 22]. Different methods for computation of a positive realization (2) for a given proper transfer matrix $T(s)$ have been proposed in $[8,13,15,22]$.

Consider the descriptor continuous-time linear system

$$
\begin{gathered}
E \dot{x}(t)=A x(t)+B u(t) \\
y(t)=C x(t)
\end{gathered}
$$

where $x(t) \in \Re^{n}, u(t) \in \mathfrak{R}^{m}, y(t) \in \mathfrak{R}^{p}$ are the state, input and output vectors and $E, A \in$ $\mathfrak{R}^{n \times n}, B \in \mathfrak{R}^{n \times m}, C \in \mathfrak{R}^{p \times n}$. It is assumed that $\operatorname{det} E=0$ and the pencil of $(E, A)$ is regular, i.e.

$$
\operatorname{det}[E s-A] \neq 0 \text { for some } s \in C \text { (the field of complex numbers). }
$$

Definition 3 The descriptor system (6) is called (internally) positive if $x(t) \in \Re_{+}^{n}$, $y(t) \in \mathfrak{R}_{+}^{p}, t \geqslant 0$ for any initial conditions $x(0)=x_{0} \in \mathfrak{R}_{+}^{n}$ and all inputs $u^{(k)}(t)=\frac{d^{k} u(t)}{d t^{k}} \in \Re_{+}^{m}, t \geqslant 0, k=0,1, \ldots, q$.

The transfer matrix of the system (6) is given by

$$
T(s)=C[E s-A]^{-1} B \in \mathfrak{R}^{p \times m}(s) .
$$

If the nilpotency index $\mu$ of the matrix $E$ is greater or equal to 1 [12] then the transfer matrix (8) is improper and can be always written as the sum of the strictly proper transfer matrix $T_{s p}(s)$ and the polynomial matrix $P(s)$, i.e.

$$
T(s)=T_{s p}(s)+P(s)
$$

where

$$
P(s)=D_{0}+D_{1} s+\ldots+D_{q} s^{q} \in \Re^{p \times m}[s], \quad q \in N=\{1,2, \ldots\}
$$


and $q=\mu-1$.

Theorem 3 [5, 13] Let the matrices

$$
A \in M_{n}, \quad B \in \mathfrak{R}_{+}^{n \times m} \quad C \in \mathfrak{R}_{+}^{p \times n}
$$

be a positive and asymptotically stable realization of the strictly proper transfer matrix $T_{s p}(s)$. Then there exists a positive asymptotically stable realization of $T(s) \in \mathfrak{R}^{p \times m}(s)$ of the form

$$
\begin{aligned}
& \bar{E}=\left[\begin{array}{cccccc}
I_{n} & 0 & 0 & \ldots & 0 & 0 \\
0 & 0 & 0 & \ldots & 0 & 0 \\
0 & I_{m} & 0 & \ldots & 0 & 0 \\
\vdots & \vdots & \vdots & \ldots & \vdots & \vdots \\
0 & 0 & 0 & \ldots & I_{m} & 0
\end{array}\right] \in \Re_{+}^{\bar{n} \times \bar{n}}, \quad \bar{A}=\left[\begin{array}{cccccc}
A & B & 0 & \ldots & 0 & 0 \\
0 & I_{m} & 0 & \ldots & 0 & 0 \\
0 & 0 & I_{m} & \ldots & 0 & 0 \\
\vdots & \vdots & \vdots & \ldots & \vdots & \vdots \\
0 & 0 & 0 & \ldots & 0 & I_{m}
\end{array}\right] \in M_{\bar{n}} \\
& \bar{B}=-\left[\begin{array}{c}
0 \\
I_{m} \\
0 \\
\vdots \\
0
\end{array}\right] \in \Re_{+}^{\bar{n} \times m}, \bar{C}=\left[\begin{array}{lllll}
C & D_{0} & D_{1} & \ldots & D_{q}
\end{array}\right] \in \mathfrak{R}_{+}^{p \times \bar{n}}, \quad \bar{n}=n+(q+1) m
\end{aligned}
$$

if and only if

$$
D_{k} \in \mathfrak{R}_{+}^{p \times m} \text { for } k=0,1, \ldots, q \text {. }
$$

Proof is given in [23].

If the conditions of Theorem 3 are satisfied then the desired positive realization (12) of $T(s)$ can be computed by the use of the following procedure [23].

\section{Procedure 1}

Step 1. Decompose the given matrix $T(s)$ into the strictly proper transfer matrix $T_{s p}(s)$ and the polynomial matrix $P(s)$ satisfying (9).

Step 2. Using one of the well-known methods [7, 13, 17-21, 22] find the positive realization (11) of $T_{s p}(s)$.

Step 3. Knowing the realization (11) and the matrices $D_{k} \in \mathfrak{R}_{+}^{p \times m}, k=0,1, \ldots, q$ of (10) find the desired realization (12).

An example illustrating the procedure has been given in [23]. 


\section{Positive fractional linear systems}

The following Caputo definition of the derivative-integral of fractional order will be used [22]

$$
{ }_{0} D_{t}^{\alpha} f(t)=\frac{d^{\alpha} f(t)}{d t^{\alpha}}=\frac{1}{\Gamma(w-\alpha)} \int_{0}^{t} \frac{f^{(w)}(\tau)}{(t-\tau)^{\alpha+1-w}} d \tau, w-1<\alpha<w, w \in N=\{1,2, \ldots\}
$$

where

$$
\Gamma(x)=\int_{0}^{\infty} t^{x-1} e^{-t} d t, \quad \operatorname{Re}(x)>0
$$

is the gamma function and

$$
f^{(w)}(\tau)=\frac{d^{w} f(\tau)}{d \tau^{w}}
$$

is the classical $w$ order derivative.

Consider the continuous-time fractional linear systems described by the state equations

$$
\begin{gathered}
\frac{d^{\alpha} x(t)}{d t^{\alpha}}=A x(t)+B u(t), 0<\alpha<1 \\
y(t)=C x(t)+D u(t)
\end{gathered}
$$

where $x(t) \in \mathfrak{R}^{n}, u(t) \in \mathfrak{R}^{m}, y(t) \in \mathfrak{R}^{p}$ are the state, input and output vectors and $A \in \mathfrak{R}^{n \times n}, B \in \mathfrak{R}^{n \times m}, C \in \mathfrak{R}^{p \times n}, D \in \mathfrak{R}^{p \times m}$.

Theorem 4 The solution of the equation (15a) satisfying the initial condition $x(0)=x_{0}$ has the form

$$
\begin{gathered}
x(t)=\Phi_{0}(t) x_{0}+\int_{0}^{t} \Phi(t-\tau) B u(\tau) d \tau \\
\Phi_{0}(t)=\sum_{k=0}^{\infty} \frac{A^{k} t^{k \alpha}}{\Gamma(k \alpha+1)}, \quad \Phi(t)=\sum_{k=0}^{\infty} \frac{A^{k} t^{(k+1) \alpha-1}}{\Gamma[(k+1) \alpha]}, \quad 0<\alpha<1 .
\end{gathered}
$$

Definition 4 [22] The fractional system (15) is called the (internally) positive fractional system if $x(t) \in \mathfrak{R}_{+}^{n}, y(t) \in \mathfrak{R}_{+}^{p}, t \geqslant 0$ for any initial conditions $x_{0} \in \mathfrak{R}_{+}^{n}$, and all inputs $u(t) \in \mathfrak{R}_{+}^{m}, t \geqslant 0$.

Let $e_{i}$ be the $i$ th column (row) of the identity matrix $I_{n}$. A column (row) of the form $a e_{i}, a>0$ is called monomial. A square matrix $A=\left[a_{i j}\right]$ is called Metzler matrix if its off-diagonal entries are nonnegative, i.e. $a_{i j} \geqslant 0$ for $i \neq j[13,5]$. The set of $n \times n$ Metzler matrices will be denoted by $M_{n}$. 
Theorem 5 [22] The fractional system (15) is (internally) positive if and only if

$$
A \in M_{n}, B \in \mathfrak{R}_{+}^{n \times m}, C \in \mathfrak{R}_{+}^{p \times n}, D \in \mathfrak{R}_{+}^{p \times m} .
$$

Consider the descriptor continuous-time linear system with regular pencil

$$
E \dot{x}(t)=A x(t)+B u(t), \quad x(0)=x_{0}
$$

where $x(t) \in \mathfrak{R}^{n}, u(t) \in \mathfrak{R}^{m}$ are the state and input vectors and $E, A \in \mathfrak{R}^{n \times n}, B \in \mathfrak{R}^{n \times m}$

$$
\operatorname{det} E=0 \text { and } \operatorname{det}[E s-A] \neq 0 \text { for some } s \in C \text { (the field of complex numbers). }
$$

Let $U_{a d}$ be a set of all given admissible inputs $u(t) \in \mathfrak{R}^{m}$ of the system (18). A set of all initial conditions $x_{0} \in \mathfrak{R}^{n}$ for which the equation (18) has a solution $x(t)$ for $u(t) \in U_{a d}$ is called the set of consistent initial conditions and is denoted by $X_{c}^{0}$. The set $X_{c}^{0}$ depends on the matrices $E, A, B$ but also on $u(t) \in U_{a d}[11]$.

Now let us consider the fractional descriptor continuous-time linear system with regular pencil satisfying (19)

$$
\begin{gathered}
E \frac{d^{\alpha} x(t)}{d t^{\alpha}}=A x(t)+B u(t), \quad x(0)=x_{0} \\
y(t)=C x(t), \quad x(0)=x_{0}
\end{gathered}
$$

where $0<\alpha<1$ is the fractional order and $x_{0} \in \mathfrak{R}^{n}, u(t) \in \mathfrak{R}^{m}$ are the state and input vectors and $E, A \in \mathfrak{R}^{n \times n}, B \in \mathfrak{R}^{n \times m}, C \in \mathfrak{R}^{p \times n}$.

Definition 5 The fractional descriptor systems (20) is called (internally) positive if $x(t) \in \mathfrak{R}_{+}^{n}, y(t) \in \mathfrak{R}_{+}^{p}, t \geqslant 0$ for every consistent nonnegative initial condition $x_{0} \in X_{c+}^{0}$ and all admissible inputs $u(t) \in U_{a d+}$, where $X_{c+}^{0}$ and $U_{a d+}$ are the sets of nonnegative consistent initial conditions and nonnegative admissible inputs, respectively.

A matrix $Q \in \Re_{+}^{n \times n}$ is called monomial if its every row and column contains only one positive entry and its remaining entries are zero. The inverse matrix $Q^{-1}$ of the monomial matrix $Q$ has only nonnegative entries, i.e. $Q^{-1} \in \Re^{n \times n_{+}}$[13].

It is assumed that using elementary row and column operations $[14,24]$ it is possible to reduce the pair $(E, A)$ to the form

$$
P[E s-A] Q=\bar{E} s-\bar{A}
$$

where

$$
\begin{aligned}
& \bar{E}=\left[\begin{array}{cc}
I_{n_{1}} & 0 \\
0 & 0
\end{array}\right], \quad \bar{A}=\left[\begin{array}{cc}
\bar{A}_{11} & \bar{A}_{12} \\
\bar{A}_{21} & \bar{A}_{22}
\end{array}\right], \\
& \bar{A}_{11} \in M_{n_{1}}, \quad \bar{A}_{22} \in M_{n_{2}}, \quad \bar{A}_{12} \in \Re_{+}^{n_{1} \times n_{2}}, \quad \bar{A}_{21} \in \Re_{+}^{n_{2} \times n_{1}},
\end{aligned}
$$


and $n_{1}=\operatorname{rank} E, n_{2}=n-n_{1} . P \in \mathfrak{R}^{n \times n}$ is a matrix of elementary row operations and $Q \in \mathfrak{R}_{+}^{n \times n}$ is a monomial matrix of elementary column operations. The matrix $P$ can be obtained by performing the elementary row operations and the matrix $Q$ by performing the elementary column operations on identity matrix $I_{n}$, respectively $[14,12]$. Note that if $Q$ is a monomial matrix then $\bar{x}(t)=Q^{-1} x(t) \in \mathfrak{R}_{+}^{n}, t \geqslant 0$ for every $x(t) \in \operatorname{Re}_{+}^{n}, t \geqslant 0$ since $Q^{-1} \in \mathfrak{R}_{+}^{n \times n}$.

Theorem 6 The descriptor continuous-time linear system (20) for $B u(t)=0$ is positive and asymptotically stable if and only if there exists a pair of elementary operations matrices $(P, Q)$ satisfying (21) such that the coefficients of the polynomials

$$
p_{1}(s)=\operatorname{det}\left[\begin{array}{cc}
I_{n_{1}} s-\bar{A}_{11} & -\bar{A}_{12} \\
-\bar{A}_{21} & -\bar{A}_{22}
\end{array}\right]=\bar{a}_{n_{1}} s^{n_{1}}+\bar{a}_{n_{1}-1} s^{n_{1}-1}+\ldots+\bar{a}_{1} s+\bar{a}_{0}
$$

and

$$
p_{2}(s)=\operatorname{det}\left[I_{n_{2}} s-\bar{A}_{22}\right]=s^{n_{2}}+a_{n_{2}-1} s^{n_{2}-1}+\ldots+a_{1} s+a_{0}
$$

are positive, i.e. $\bar{a}_{j}>0, j=0,1, \ldots, n_{1}$ and $a_{i}>0, i=0,1, \ldots, n_{2}-1$.

Proof is given in [24].

\section{Realization problem for positive fractional linear systems}

The transfer matrix of the fractional descriptor continuous-time linear system with regular pencil is given by [22]

$$
T(\lambda)=C[E \lambda-A]^{-1} B \in \mathfrak{R}^{p \times m}(\lambda) \quad\left(\lambda=s^{\alpha}\right)
$$

The improper transfer matrix (23) can be decomposed into strictly proper transfer matrix $T_{s p}(\lambda)$ and the polynomial matrix $P(\lambda)$, i.e.

$$
T(\lambda)=T_{s p}(\lambda)+P(\lambda)
$$

where

$$
P(\lambda)=D_{0}+D_{1} \lambda+\ldots+D_{q} \lambda^{q} \in \Re^{p \times m}[\lambda], \quad q=\mu-1
$$

and $\mu$ is the nilpotency index.

Using one of the well-known methods [7, 13, 17, 21, 22] we may find a positive asymptotically stable realization (11) of $T_{s p}(\lambda)$ and from (12) the desired positive asymptotically stable realization of $T(\lambda)$. Therefore, Procedure 1 can be applied to compute the desired positive asymptotically stable realization (12) of $T(\lambda)$ by replacing $s^{\alpha}$ by $\lambda$. 
Example 1. Find a positive asymptotically stable realization (12) of the transfer matrix

$$
T(\lambda)=\left[\begin{array}{cc}
\frac{2 \lambda^{3}+5 \lambda^{2}+5 \lambda+4}{\lambda+1} & \frac{3 \lambda^{2}+7 \lambda+3}{\lambda+2} \\
\frac{\lambda^{3}+2 \lambda^{2}+2 \lambda+5}{\lambda+2} & \frac{\lambda^{3}+5 \lambda^{2}+7 \lambda+4}{\lambda+3}
\end{array}\right] .
$$

Using Procedure 1 we obtain the following.

Step 1. The transfer matrix (25) has the strictly proper transfer matrix

$$
T_{s p}(\lambda)=\left[\begin{array}{ll}
\frac{2}{\lambda+1} & \frac{1}{\lambda+2} \\
\frac{1}{\lambda+2} & \frac{1}{\lambda+3}
\end{array}\right]
$$

and the polynomial matrix

$$
P(\lambda)=\left[\begin{array}{cc}
2 \lambda^{2}+3 \lambda+2 & 3 \lambda+1 \\
\lambda^{2}+2 & \lambda^{2}+2 \lambda+1
\end{array}\right]=D_{0}+D_{1} \lambda+D_{2} \lambda^{2}, \quad(q=2)
$$

where

$$
D_{0}=\left[\begin{array}{ll}
2 & 1 \\
2 & 1
\end{array}\right], \quad D_{1}=\left[\begin{array}{ll}
3 & 3 \\
0 & 2
\end{array}\right], \quad D_{2}=\left[\begin{array}{ll}
2 & 0 \\
1 & 1
\end{array}\right]
$$

Step 2. The strictly proper transfer matrix (26) can be rewritten in the form

$$
T_{s p}(\lambda)=\frac{1}{(\lambda+1)(\lambda+2)(\lambda+3)}\left[\begin{array}{cc}
2(\lambda+2)(\lambda+3) & (\lambda+1)(\lambda+3) \\
(\lambda+1)(\lambda+3) & (\lambda+2)(\lambda+1)
\end{array}\right]
$$

and the well-known Gilbert method can be applied to find its positive asymptotically stable realization $[7,13,25]$ since the poles of (28) are distinct and negative $\left(\lambda_{1}=-1, \lambda_{2}=-2, \lambda_{3}=-3\right)$. Following the Gilbert method we compute the matrices

$$
\begin{aligned}
& T_{1}=\frac{1}{(\lambda+2)(\lambda+3)}\left[\begin{array}{cc}
2(\lambda+2)(\lambda+3) & (\lambda+1)(\lambda+3) \\
(\lambda+1)(\lambda+3) & (\lambda+2)(\lambda+1)
\end{array}\right]_{\lambda=-1}=\left[\begin{array}{ll}
2 & 0 \\
0 & 0
\end{array}\right], \\
& r_{1}=\operatorname{rank} T_{1}=1, \quad T_{1}=C_{1} B_{1}, \quad C_{1}=\left[\begin{array}{l}
2 \\
0
\end{array}\right], \quad B_{1}=\left[\begin{array}{ll}
1 & 0
\end{array}\right], \\
& T_{2}=\frac{1}{(\lambda+1)(\lambda+3)}\left[\begin{array}{cc}
2(\lambda+2)(\lambda+3) & (\lambda+1)(\lambda+3) \\
(\lambda+1)(\lambda+3) & (\lambda+2)(\lambda+1)
\end{array}\right]_{\lambda=-2}=\left[\begin{array}{ll}
0 & 1 \\
1 & 0
\end{array}\right],
\end{aligned}
$$




$$
\begin{aligned}
& r_{2}=\operatorname{rank} T_{2}=2, \quad T_{2}=C_{2} B_{2}, \quad C_{2}=\left[\begin{array}{ll}
1 & 0 \\
0 & 1
\end{array}\right], \quad B_{2}=\left[\begin{array}{ll}
0 & 1 \\
1 & 0
\end{array}\right], \\
& T_{3}=\frac{1}{(\lambda+1)(\lambda+2)}\left[\begin{array}{cc}
2(\lambda+2)(\lambda+3) & (\lambda+1)(\lambda+3) \\
(\lambda+1)(\lambda+3) & (\lambda+2)(\lambda+1)
\end{array}\right],=\left[\begin{array}{ll}
0 & 0 \\
0 & 1
\end{array}\right], \\
& r_{3}=\operatorname{rank} T_{3}=1, \quad T_{3}=C_{3} B_{3}, \quad C_{3}=\left[\begin{array}{l}
0 \\
1
\end{array}\right], \quad B_{3}=\left[\begin{array}{ll}
0 & 1
\end{array}\right] .
\end{aligned}
$$

Thus, the positive asymptotically stable realization of (26) has the form

$$
\begin{aligned}
& A=\operatorname{block} \operatorname{diag}\left[I_{r_{1}} \lambda_{1}, I_{r_{2}} \lambda_{2}, I_{r_{3}} \lambda_{3}\right]=\operatorname{diag}[-1,-2,-2,-3], \\
& B=\left[\begin{array}{l}
B_{1} \\
B_{2} \\
B_{3}
\end{array}\right]=\left[\begin{array}{ll}
1 & 0 \\
0 & 1 \\
1 & 0 \\
0 & 1
\end{array}\right], \quad C=\left[\begin{array}{lll}
C_{1} & C_{2} & C_{3}
\end{array}\right]=\left[\begin{array}{llll}
2 & 1 & 0 & 0 \\
0 & 0 & 1 & 1
\end{array}\right] .
\end{aligned}
$$

Step 3. Using (12), (27) and (29) we obtain the desired positive asymptotically stable realization of (25) in the form

$$
\bar{E}=\left[\begin{array}{cccc}
I_{4} & 0 & 0 & 0 \\
0 & 0 & 0 & 0 \\
0 & I_{2} & 0 & 0 \\
0 & 0 & I_{2} & 0
\end{array}\right] \in \mathfrak{R}_{+}^{\bar{n} \times \bar{n}}, \bar{A}=\left[\begin{array}{cccc}
A & B & 0 & 0 \\
0 & I_{2} & 0 & 0 \\
0 & 0 & I_{2} & 0 \\
0 & 0 & 0 & I_{2}
\end{array}\right] \in M_{n}, \quad \bar{B}=\left[\begin{array}{c}
0 \\
-I_{2} \\
0 \\
0
\end{array}\right] \in \Re_{+}^{\bar{n} \times 2},
$$

$\bar{C}=\left[\begin{array}{llll}C & D_{0} & D_{1} & D_{2}\end{array}\right] \in \mathfrak{R}_{+}^{2 \times \bar{n}}, \quad \bar{n}=n+(q+1) m=4+3 \cdot 2=10$.

and the matrices $A, B, C, D_{0}, D_{1}, D_{2}$ are given by (30) and (27b).

\section{Concluding remarks}

The method for computation of positive asymptotically stable realizations of fractional descriptor continuous-time linear systems with regular pencils has been proposed. The method is based on the decomposition of the given improper transfer matrix $T(\lambda)$. The desired positive asymptotically stable realizations of $T(\lambda)$ can be computed by the use of Procedure 1. The method has been illustrated by the example. The method can be extended to fractional descriptor discrete-time linear systems. An open problem is an extension of the method to fractional descriptor continuous-discrete linear systems. 


\section{References}

[1] L. Benvenuti and L. FARINA: A tutorial on the positive realization problem. IEEE Trans. on Automatic Control, 49(5), (2004), 651-664.

[2] L. DAI: Singular control systems. Lecture Notes in Control and Inform. Sci. 118 Springer-Verlag, Berlin, 1989.

[3] R. Bru, C. Coll, S. Romero-Vivo and E. Sanchez: Some problems about structural properties of positive descriptor systems. Positive systems (Rome, 2003), Lecture Notes in Control and Inform. Sci. 294 Springer, Berlin, 2003, 233-240.

[4] R. BRU, C. Coll and E. SANCheZ: Structural properties of positive linear timeinvariant difference-algebraic equations. Linear Algebra Appl., 349 (2002), 1-10.

[5] L. FARINA and S. RinAldi: Positive Linear Systems; Theory and Applications. J. Wiley, New York, 2000.

[6] T. KACZOREK: A realization problem for positive continuous-time linear systems with reduced numbers of delays. Int. J. Appl. Math. Comp. Sci., 16(3), (2006), 325-331.

[7] T. KACZOREK: Computation of positive stable realization for linear continuoustime systems. Bull. Pol. Acad. Sci. Techn., 59(3), (2011), 273-281.

[8] T. KaczoreK: Computation of realizations of discrete-time cone systems. Bull. Pol. Acad. Sci. Techn., 54(3), (2006), 347-350.

[9] T. KACZOREK: Checking of the positivity of descriptor linear systems by the use of the shuffle algorithm. Archives of Control Sciences, 21(3), (2011), 287-298.

[10] T. KACZOREK: Fractional positive continuous-time linear systems and their reachability. Int. J. Appl. Math. Comp. Sci., 18(2), (2008), 223-228.

[11] T. KaczoreK: Fractional positive linear systems. Kybernetes: The International Journal of Systems and Cybernetics, 38(7/8), (2009), 1059-1078.

[12] T. Kaczorek: Linear Control Systems. 1 Research Studies Press, J. Wiley, New York 1992.

[13] T. KaCzoreK: Positive 1D and 2D Systems. Springer-Verlag, London, 2002.

[14] T. Kaczorek: Polynomial and Rational Matrices. Springer-Verlag, London, 2009.

[15] T. KaCzoreK: Positive stable realizations of fractional continuous-time linear systems. Int. J. Appl. Math. Comp. Sci., 21(4), (2011), 697-703. 
[16] T. KaczoreK: Positive minimal realizations for singular discrete-time systems with delays in state and delays in control. Bull. Pol. Acad. Sci. Techn., 53(3), (2005), 293-298.

[17] T. Kaczorek: Positive stable realizations with system Metzler matrices. Proc. MMAR Conf., 22-25 Sept. 2011, Miédzyzdroje, Poland.

[18] T. KACZOREK: Realization problem for fractional continuous-time systems. Archives of Control Sciences, 18(1), (2008), 43-58.

[19] T. KaCzOREK: Realization problem for positive 2D hybrid systems. COMPEL, 27(3), (2008), 613-623.

[20] T. KACZOREK: Realization problem for positive multivariable discrete-time linear systems with delays in the state vector and inputs. Int. J. Appl. Math. Comp. Sci., 16(2), (2006), 101-106.

[21] T. KACZOREK: Realization problem for positive discrete-time systems with delays. System Science, 30(4), (2004), 117-130.

[22] T. KaCzoreK: Selected Problems in Fractional Systems Theory. Springer-Verlag, 2011

[23] T. KACZOREK: Positive realizations for descriptor continuous-time linear systems. $P A K$, (2012).

[24] T. KaCZOREK: Stability of descriptor positive linear systems. COMPEL, (2012), (in press).

[25] T. KaczoreK: Singular fractional linear systems and electrical circuits. Int. J. Apll. Math. Comput. Sci., 21(2), (2011), 379-384.

[26] E. VIRNIK: Stability analusis of positive descriptor systems. Linear Algebra and its Applications, 429 (2008), 2640-2659.

[27] U. SHAKER and M. DIXON: Generalized minimal realization of transfer-function matrices. Int. J. Contr., 25(5), (1977), 785-803. 